\title{
The Effect of ZSM-5 Zeolite Crystal Size on p-xylene Selectivity in Toluene Disproportionation
}

\author{
Mohammed Albahar ${ }^{*}$, Chaozhou Li ${ }^{b}$, Vladimir L. Zholobenko ${ }^{c}$, Arthur A. Garforth ${ }^{b}$ \\ a \\ Research and Development Center, Saudi Aramco, Dhahran 31311, Saudi Arabia, *corresponding author b \\ School of Chemical Engineering and Analytical science, The University of Manchester, UK, M13 9PL c \\ School of Chemical and Physical Sciences, Keele University, UK
}

\begin{abstract}
The effect of crystal size was explored in this work aiming at enhancing p-xylene selectivity through toluene disproportionation over ZSM-5 zeolite. The different physicochemical properties of ZSM-5 were investigated using various characterization techniques including $\mathrm{X}$-ray diffraction (XRD), pyridine adsorption, Fourier transform infra-red (FTIR), BET surface area by $\mathrm{N}_{2}$ adsorption, inductively coupled plasma (ICP) and scanning electron microscopy (SEM). Each catalyst was tested in a fixed bed reactor at a temperature $475^{\circ} \mathrm{C}$, weight hourly space velocity (WHSV) 3-83 $\mathrm{h}^{-1}$ and two different pressures (1 and 10 bar). ZSM-5 zeolites with crystal sizes 5, 50 and $100 \mu \mathrm{m}$ were synthesized in house and compared with the commercially obtained ZSM- 5 having a crystal size of $0.5 \mu \mathrm{m}$. As a result of increasing the crystal size the p-xylene selectivity was improved. This was attributed to the longer diffusion path lengths of the large crystals which imposed more diffusion constraints on the other xylene isomers. ZSM-5 zeolite with the largest crystal size $100 \mu \mathrm{m}$ achieved the highest p-xylene selectivity (58\%) at the highest WHSV $83 \mathrm{~h}^{-1}$. However, this was accompanied by a low conversion ( $2 \mathrm{wt}$. \%). The best combination of p-xylene selectivity (40\%) alongside 15 wt. \% toluene conversion were achieved over ZSM-5 having $5 \mu \mathrm{m}$ crystal sized.
\end{abstract}

Keywords: ZSM-5 Zeolite, Toluene, disproportionation, selectivity, Para-xylene

\section{Introduction}

Para-xylene is a highly demanded raw material mainly utilized in the synthesis of terephthalic acid, dimethyl terephthalate and purified terephthalic acid. These compounds are 
used to make polyesters such as polyethylene terephthalate (PET) which are widely used for making carbonated drinks and water containers [1,2]. As a result of the increasing demand on $p$ xylene in recent years (6-8\% per year), the consumption will surpass the supply in the next few years [3]. To meet the increasing demand, the supply of $p$-xylene has to be increased in order to meet the high increasing demand. However, most of the plants are operating at full capacity. The production of $p$-xylene can be enhanced by converting less demanded and valuable refinery products, such as toluene through the disproportionation reaction to more demanded products like benzene and xylene. However, carrying out toluene disproportionation over commercial zeolites yields the thermodynamic equilibrium mixture of xylene isomers, approximately $23 \% \mathrm{p}$ xylene, 51\% m- and 26\% o-xylene) [4]. In the 1980s, Mobil developed ZSM-5 zeolite catalyst that has 10 membered rings channel system and pore size $(0.51-0.58 \mathrm{~nm})$. The structure of the catalyst offered an excellent performance as the pore opening almost matches the molecular diameters size of the main products and reactants (toluene, benzene and xylenes). Moreover, the diffusion of para-xylene in ZSM-5 is favoured over ortho- and meta-xylenes as it has the smallest molecular diameter compared to the other isomers. This is a significant factor making ZSM-5 stand out from other zeolite catalysts in terms of performance in toluene disproportionation $[5,6]$. Although it is anticipated that $p$-xylene is a primary product leaving the pore mouth of ZSM-5 zeolite, it isomerizes on the external surface which leads to the production of xylene isomers in their thermodynamic equilibrium values. To obtain higher $p$-xylene selectivity than the thermodynamic equilibrium value, it was suggested that deactivating the external acid sites of the catalyst and increasing the diffusion pathway by increasing the crystal size are crucial factors [4, 7-16]. A unique relationship between o-xylene diffusion time, by adsorption-measurements, and p-xylene selectivity was found by Olson and Haag. They noticed 
that increasing the crystal size of the zeolite reduced the diffusion rate and o-xylene diffusion.

Therefore, $p$-xylene selectivity can be improved [17].

Different studies in the literature reported that intracrystalline diffusion limitations can have a major effect on catalyst performance. Moreover, reducing or increasing the zeolite crystal size results in noticeable changes in the reaction rate and product selectivity $[18,19]$. Hodala et al. [20] found that the catalyst crystal size correlates directly with selectivity towards para isomer where employing larger crystals during ethylbenzene alkylation with ethanol delivered higher para-diethylbenzene selectivity than the thermodynamic equilibrium value. The diffusion rate of the desired para-xylene isomer inside the channels is much higher than ortho- and meta-xylenes. Thus, increasing the crystal size will lead to obtaining more $p$-xylene in the product stream as the other isomers will have to isomerize to the much faster diffusing isomer ( $p$-xylene) in order to diffuse out of the channels [21, 22]. Ratnasamy et al. [10] performed xylene isomerization reaction over ZSM-5 zeolites with different crystal size to investigate the effects of diffusion path length on the activity and selectivity towards p-xylene. ZSM-5 catalysts with 8,13 and $16 \mu \mathrm{m}$ were tested and it was clearly shown that the activity was decrease decreased by a factor of 3 as a result of increasing the crystal size from 8 to $16 \mu \mathrm{m}$. Conversely, the ratio of $\mathrm{p}$-xylene to oxylene increased from 1.2 to 2.5 and hence more p-xylene formed. Also, alkylation of toluene with methanol over ZSM-5 zeolites with 0.1 and $7 \mu \mathrm{m}$ crystal size having Si/Al ratio of 70 was carried out by Chen et al [23]. P-xylene selectivity was enhanced reaching $50 \%$ over the large crystals and the thermodynamic equilibrium value of pxylene (24\%) was obtained over the small crystals.

In this study, ZSM-5 zeolite with various crystal sizes were employed to study the effect of diffusion path on toluene disproportionation and aiming to improve the selectivity towards $p$ xylene. Furthermore, the effect of residence time and pressure on the reaction were investigated. 


\section{Experimental}

\subsection{Catalyst preparation}

ZSM-5 zeolite with different crystal sizes $(5,50$ and $100 \mu \mathrm{m})$ were hydrothermally synthesized and a commercial ZSM-5 with $0.5 \mu \mathrm{m}$ crystal size with Si/Al ratio of 25 was obtained from Alfa Aesar.

\subsubsection{ZSM-5 with 5 um crystal size}

Feedstock and seeding gel were prepared to synthesize and produce NaZSM-5 having a Si/Al=16 and $5 \mu \mathrm{m}$ crystals [24]. Colloidal silica (Ludox AS-40), sodium Hydroxide, tetrapropylammonium hydroxide (TPAOH) and deionized water to prepare the seeding gel according to the gel molar ratio $\mathrm{Na}_{2} \mathrm{O}: 3 \mathrm{TPAOH}: 60 \mathrm{SiO}_{2}: 1200 \mathrm{H}_{2} \mathrm{O}$. The seeding gel was aged overnight at $100{ }^{\circ} \mathrm{C}$. Preparing the feedstock gel was done by mixing sodium aluminate $\left(\mathrm{NaAlO}_{2}\right)$, sodium hydroxide $(\mathrm{NaOH})$ and colloidal silica in deionized water according to the gel composition $6 \mathrm{Na}_{2} \mathrm{O}: 2 \mathrm{Al}_{2} \mathrm{O}_{3}: 60 \mathrm{SiO}_{2}: 1916 \mathrm{H}_{2} \mathrm{O}$. The seeding gel was mixed with the feedstock gel and poured into a PTFE-lined autoclave. The autoclave was placed in an oven at $180^{\circ} \mathrm{C}$ for

$24 \mathrm{hrs}$. The autoclave was then cooled, and the obtained solid was washed, filtered and dried at $120^{\circ} \mathrm{C}$. Then, the powder was calcined at $550^{\circ} \mathrm{C}$ for 8 hours to remove the template. After that, the zeolite was ion exchanged with ammonium nitrate solution to remove the sodium. Finally, the ammonium form of ZSM-5 was calcined in air at $550^{\circ} \mathrm{C}$ for 8 hours to produce $\mathrm{H}-\mathrm{ZSM}-5$.

\subsubsection{ZSM-5 with 50 and $100 \mu \mathrm{m}$ crystal size}

Following the reported procedure by Chen et al. [25], the synthesis was performed in a PTFE-lined autoclave over 10 days at $170{ }^{\circ} \mathrm{C}$ for to produce ZSM-5 zeolite with $50 \mu \mathrm{m}$ crystals. Colloidal silica (Ludox AS-40), $\mathrm{Al}(\mathrm{OH})_{3}$, tetrapropylammonium bromide (TPABr), $\mathrm{NaOH}$, potassium hydroxide $(\mathrm{KOH})$, ammonium carbonate $\left[\mathrm{NH}_{4} \mathrm{HCO}_{3} . \mathrm{NH}_{2} \mathrm{COONH}_{4}\right]$ and distilled water were used to form the synthesis gel. Firstly, the $\mathrm{NaAlO}_{2}$ solution was obtained by mixing the required 
amount of $\mathrm{Al}(\mathrm{OH})_{3}$ with $\mathrm{NaOH}$ and distilled water which then poured in an autoclave and placed

in an oven at $170{ }^{\circ} \mathrm{C}$ for 16 hours. $\mathrm{NaOH}$ and $\mathrm{KOH}$ were dissolved in $80 \%$ of the water amount required for the synthesis. Ludox was added drop wise to this solution and kept stirring for 1 hour. The $\mathrm{NaAlO}_{2}$ solution was added to $10 \%$ of the amount of water used in this synthesis and the remaining $10 \%$ of water was used to dilute the ammonium carbonate. After that, TPABr, $\mathrm{NaAlO}_{2}$ and ammonium carbonate were added to the silicate solution. The obtained gel with molar ratios (51 SiO $2: \mathrm{NaAlO}_{2}: 9 \mathrm{NaOH}: 0.5 \mathrm{KOH}: 12 \mathrm{TPABr}: 9 \mathrm{NH}_{4} \mathrm{HCO}_{3} \mathrm{NH}_{2} \mathrm{COONH}_{4}: 1300 \mathrm{H}_{2} \mathrm{O}$ ) was aged overnight at room temperature. Then, it was transferred to an autoclave and placed in the oven at $170{ }^{\circ} \mathrm{C}$ under autogenous pressure for 10 days. The final product was filtered, washed with distilled water, dried and then calcined in air at $550{ }^{\circ} \mathrm{C}$ for 8 hours. Following the same procedure, the $100 \mu \mathrm{m}$ crystals were generated using but instead the autoclave was placed in the oven for 12 days which resulted in larger crystals.

\subsection{Catalyst characterization}

The physical and chemical properties of the synthesized and commercially obtained zeolite samples were investigated using different characterization techniques. The X-ray patterns and erystallinity crystallinities of the ZSM-5 Zeolite samples were acquired using Philips X'Pert Pro PW 3719 diffractometer. The data was collected at $2 \theta$ from $5^{\circ}$ to $90^{\circ}$ and a step size of $0.0407^{\circ}$ and a step time of 64 seconds. The instrument was operated at a $40 \mathrm{KV}$ tension and a current of $30 \mathrm{~mA}$. To study the morphology and size of synthesized zeolite crystals, scaning electron microscopy (SEM) (Philips XL30 FEGSEM) was utilized. Measuring the acidity of the catalyst is a crucial task in understanding the catalytic behavior of the zeolite. It is important to quantify both Brønsted acid sites (BAS) and Lewis acid sites (LAS) as they have different acidic behaviors. The study was performed by collecting and analyzing FTIR spectra using a Thermo iS10 spectrometer accumulating 64 scans at $4 \mathrm{~cm}^{-1}$ resolution. Firstly, the zeolite samples were pressed into self- 
supported discs and activated under vacuum at a rate of $1 / \mathrm{min}$ up to $450^{\circ} \mathrm{C}$ and held for 5 hours.

Pyridine (py) was used as a probe molecule to monitor the quantity of both BAS and LAS. It was introduced to the transmittance cell to saturate the samples at $150^{\circ} \mathrm{C}$ in a stepwise manner. The saturated samples were then evacuated at $150^{\circ} \mathrm{C}$ to remove any physically adsorbed pyridine and the FTIR spectrum collected. The observable peaks at $~ 1540$ and $1450 \mathrm{~cm}^{-1}$ represents py-BAS and py-LAS, respectively. The intensity of the peaks were determined to compare the number of active sites in the different samples. BET surface areas were measured at $-196{ }^{\circ} \mathrm{C}$ by nitrogen adsorption using Micromeritics Gemini 2365 surface area analyzer. Also, pore volumes were obtained. The Si/Al ratios were determined by firstly dissolving the samples in Hydrofluric acid. Then, inductively coupled plasma optical emission spectroscopy (ICP-OES) analysis were carried out. Furthermore, the Coke content on the samples after the reaction were measured using thermogravimetric analysis (TGA- Q5000IR TA). The coke amount deposited on the catalyst during the reaction was obtained by calculating the difference between the weight before and after calcination in air.

\subsection{Catalyst evaluation with toluene disproportionation}

Toluene disproportionation was performed in a fixed bed reactor attached to a condenser. The reaction was carried out over the in-house synthesized and the commercially obtained ZSM-5 zeolites. The catalyst powder was pelleted and sieved to the desired particle size $(250-425 \mu \mathrm{m})$ and $1 \mathrm{~g}$ was loaded in the reactor. Before starting the reaction, all catalysts were calcined in situ at $550^{\circ} \mathrm{C}$ for 4 hours at a heating rate of $2^{\circ} \mathrm{C} \mathrm{min}^{-1}$ in air. The reaction temperature was set at $475^{\circ} \mathrm{C}$ and the toluene weight hourly space velocity (WHSV) was varied (3-100) $\mathrm{h}^{-1}$. Moreover, the effect of pressure (1 and 10) bar on toluene conversion and p-xylene selectivity was investigated. Hydrogen was fed as a carrier gas at a constant flowrate of $30 \mathrm{ml} / \mathrm{min}$. Liquid samples were analyzed by Varian 3400 GC equipped with a $30 \mathrm{~m} \times 0.1 \mu \mathrm{m} \times 0.32 \mathrm{~mm}$ Stabilwax 
were calculated using the below formulae:

$$
\begin{aligned}
& X_{T}=\frac{W_{T 0}-W_{T}}{W_{T 0}} \times 100 \\
& S_{P}=\frac{W_{P X}}{W_{X}} \times 100
\end{aligned}
$$

Where $\mathrm{W}_{T 0}$ is the weight of toluene in the feed, $\mathrm{W}_{\mathrm{T}}$ is the weight of toluene in the product, WPX is the weight of $p$-xylene and $W_{x}$ is the weight of total xylene.

\section{Results and discussion}

\subsection{Catalyst characterization}

The generated XRD patterns are shown in Figure 1. They indicated that all samples exhibited good crystallinity and the commercial ZSM-5 showed the least intense diffraction peaks. It can be noticed in Figure 2 that SEM images that all four ZSM- 5 catalysts have different morphologies with crystal sizes ranging from 0.5 to $100 \mu \mathrm{m}$. Agglomerates of irregularly shaped crystals were observed in the case of the commercial ZSM- 5 and the synthesized $5 \mu \mathrm{m}$ showed spherical aggregates with an outer surface that consisted of stacked cubic crystallites. On the other hand, the synthesized zeolite with 50 and $100 \mu \mathrm{m}$ displayed elongated hexagonal crystals. The difference in the morphology of the crystals can be attributed to the difference in the applied synthesis parameters, including synthesis time and gel composition, used to produce ZSM-5 zeolites. 


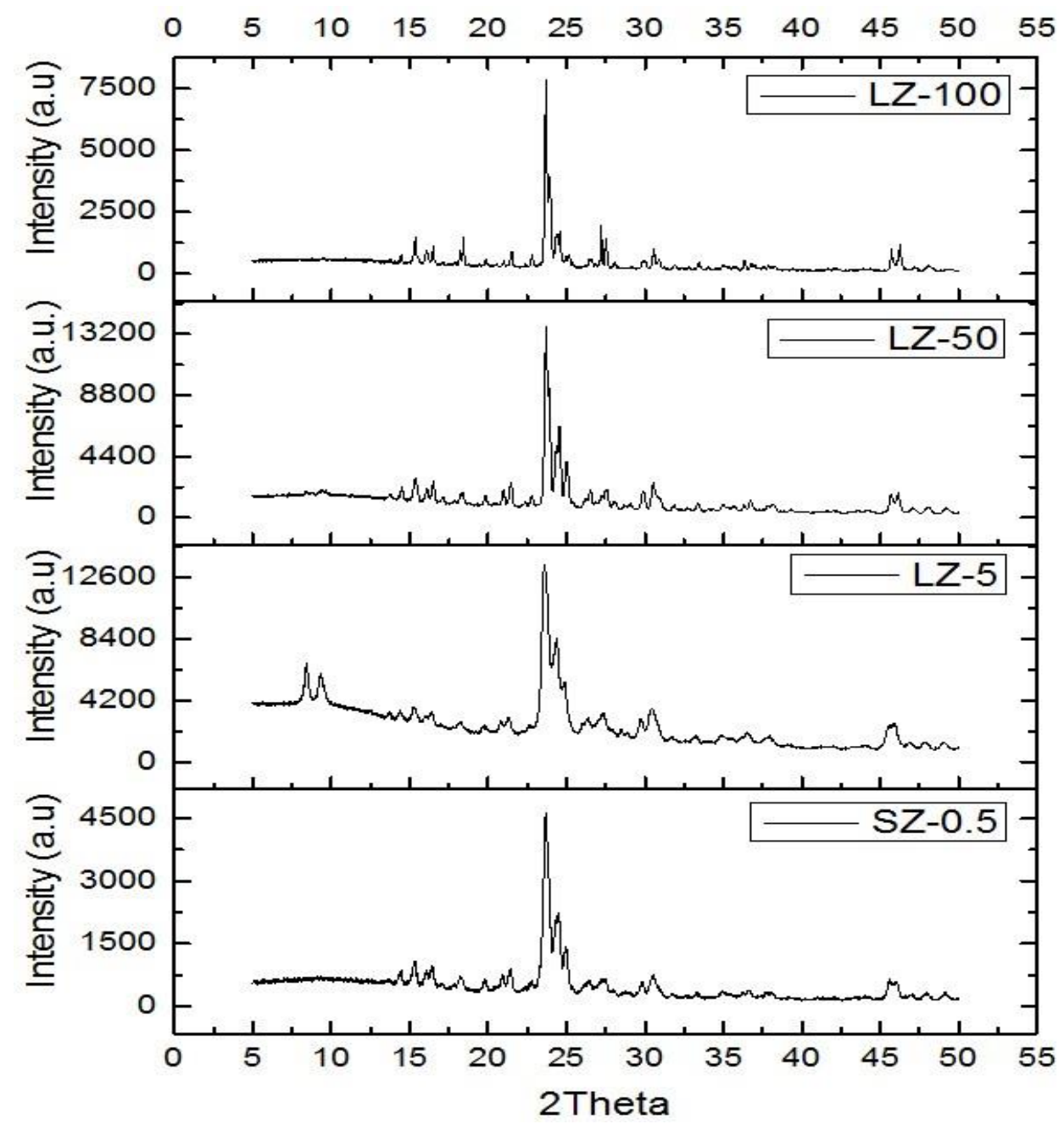

Figure 1: XRD patterns for different ZSM-5 zeolites with varying crystal size 


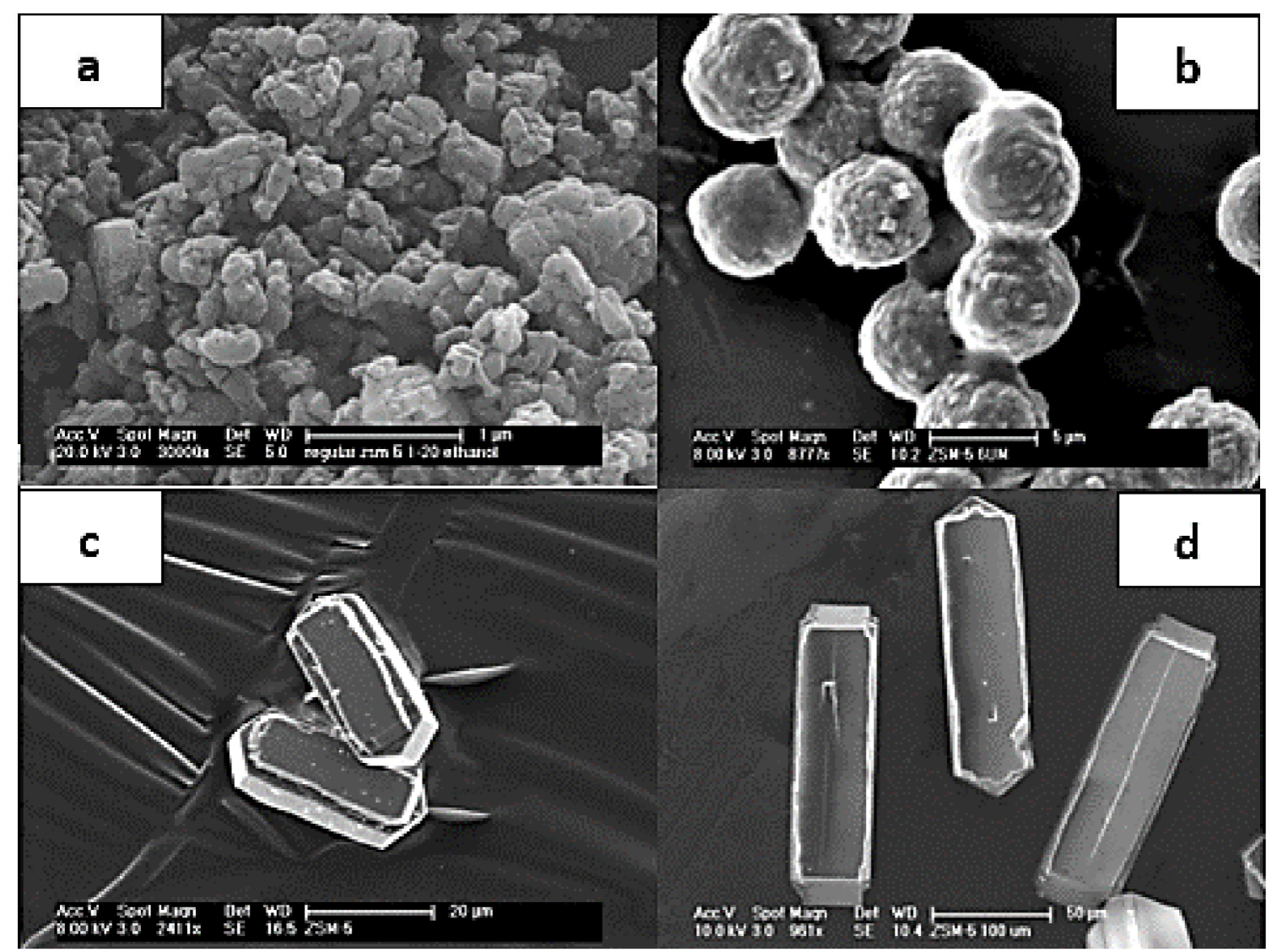

Figure 2: ZSM-5 with different crystal sizes (a) $0.5 \mu \mathrm{m}$ (b) $5 \mu \mathrm{m}$ (c) $50 \mu \mathrm{m}$ (d) $100 \mu \mathrm{m}$

The obtained Si/Al ratios by ICP-OES analyses are shown in Table 1 where it can be seen that ZSM- 5 with $5 \mu \mathrm{m}$ crystals had the lowest Si/AI ratio. FTIR spectra were collected with and without pyridine. The bridging hydroxyl groups $\left(3610 \mathrm{~cm}^{-1}\right)$ and the SiOH $\left(3745 \mathrm{~cm}^{-1}\right)$ are shown in the region $3500-3900 \mathrm{~cm}^{-1}$. As seen in Figure 4, pyridine accessed all the bridging OHgroups of the ZSM-5 zeolites with different crystal sizes. The highest number of bridging hydroxyl groups was attained by the ZSM-5 zeolite having $5 \mu \mathrm{m}$ crystal size (Si/Al=16). Brønsted and Lewis peaks were found at 1455 and $1545 \mathrm{~cm}^{-1}$, respectively after pyridine adsorption. As expected, it is noticeable in Figure 5 that ZSM-5 with $5 \mu \mathrm{m}$ crystals has the highest number of Brønsted and Lewis acid sites as it has the highest aluminum content and lowest Si/Al.

Conversely, the other catalysts have lower acidity as they contain lower aluminum. In terms of the ratio of BAS to LAS, all the catalysts differ from each other's and LZ-5 had the highest amount of LAS which could mean higher amount of extraframework aluminum as can be seen in Table 1. 
The measured level of acidity from FTIR correlated well with the Si/AI (from ICP-OES) as the zeolite with the highest Si/Al has the lowest total acidity (Brønsted + Lewis). It was realized during the synthesis processes of the very large crystals $(50,100 \mu \mathrm{m})$ that not all the aluminum is incorporated. Also, it was indicated in the literature by Muller et al that during the synthesis of large crystals, aluminum is incorporated in the framework toward the end of the synthesis resulting in higher aluminum content on the edges of the hexagonal elongated crystals [27]. All this contributes to high $\mathrm{Si} / \mathrm{Al}$ achieved during the synthesis of the very large crystals.

Table 1: ZSM-5 Zeolites Si/Al and Acidity measurements

\begin{tabular}{lcccccccc}
\hline Catalyst & $\begin{array}{c}\text { Crystal } \\
\text { size }(\mu \mathrm{m})\end{array}$ & $\begin{array}{c}\text { Si/Al } \\
(\mathrm{ICP})\end{array}$ & $\begin{array}{c}\text { BAS } \\
(\mathrm{mmol} / \mathrm{g})\end{array}$ & $\begin{array}{c}\text { LAS } \\
(\mathrm{mmol} / \mathrm{g})\end{array}$ & $\begin{array}{c}\text { BET surface } \\
\text { area }\left(\mathrm{m}^{2} / \mathrm{g}\right)\end{array}$ & $\begin{array}{c}\text { Microporous } \\
\text { area }\left(\mathrm{m}^{2} / \mathrm{g}\right)\end{array}$ & $\begin{array}{c}\text { External } \\
\text { surface area } \\
\left(\mathrm{m}^{2} / \mathrm{g}\right)\end{array}$ & $\begin{array}{c}\text { Pore volume } \\
\left(\mathrm{cm}^{3} / \mathrm{g}\right)\end{array}$ \\
\hline SZ-0.5 & 0.5 & 26.3 & 0.31 & 0.08 & 338 & 188 & 150 & 0.113 \\
LZ-5 & 5 & 15.9 & 0.37 & 0.17 & 280 & 187 & 93 & 0.110 \\
LZ-50 & 50 & 58.2 & 0.18 & 0.06 & 320 & 139 & 181 & 0.090 \\
LZ-100 & 100 & 64.3 & 0.15 & 0.05 & 309 & 137 & 173 & 0.088 \\
\hline
\end{tabular}

Nitrogen adsorption was utilized to measure the surface area of the commercial and synthesized ZSM-5 zeolites. Furthermore, the pore volumes and external surface areas were obtained. The results displayed that the highest surface area was attained by ZSM-5 with the smallest crystal size $(0.5 \mu \mathrm{m})$ while unexpectedly the zeolite with crystal size $5 \mu \mathrm{m}$ had the lowest surface area amongst all samples (Table 1) which might be related to the spherical particle shape. It can also be observed that LZ-5 had lower BET surface area $\left(280 \mathrm{~m}^{2} / \mathrm{g}\right)$ and external surface area $\left(93 \mathrm{~m}^{2} / \mathrm{g}\right)$ area as a result of the different morphology compared to the other samples. The large elongated hexagonal crystals showed higher BET surface area and external surface area which are comparable to the smallest crystals LZ-0.5. Furthermore, ZSM-5 with the largest crystal size $(100 \mu \mathrm{m})$ showed the lowest pore volume value $\left(0.088 \mathrm{~cm}^{3} / \mathrm{g}\right)$. 


\subsection{Toluene disproportionation reaction}

Carrying out toluene disproportionation reaction over ZSM-5 zeolites with different crystal sizes mainly produces benzene and a mixture of para-, meta-, ortho- xylenes. Other products with minor amounts including trimethylbenzenes and ethylbenzene are also formed.

\subsubsection{Effect of crystal size on p-xylene selectivity and toluene conversion}

The performance and stability of all catalysts were tested for two hours at $3 \mathrm{~h}^{-1}$ WHSV. It was anticipated that increasing the crystal size will improve the selectivity towards $p$-xylene because of the imposed diffusion limitations on the other xylene isomer by the longer diffusion path inside the channels. Both ZSM- 5 zeolites with 0.5 and $5 \mu \mathrm{m}$ delivered similar conversion and para-selectivity around $25 \%$ in line with the thermodynamic equilibrium value. As expected, the para-selectivity was clearly improved over the much larger crystals of ZSM-5 (50 and $100 \mu \mathrm{m}$ ). They delivered a value above the thermodynamic equilibrium value where both catalysts reached about $35 \%$ p-xylene selectivity. However, the conversion was low which was attributed to catalysts low acidity and diffusion limitation caused by the long diffusion pathways. This can be seen in Table 1 where the large crystals having half the the Brønsted and Lewis acidity of the 0.5 $\mu \mathrm{m}$ commercial ZSM-5. It is well established in zeolites that the catalytic properties of zeolites are affected by the aluminium content and distribution in the framework which is directly related to the number of acid sites [26]. It was expected that LZ-50 and LZ-100 were going to provide low toluene conversion due to the fewer available acid sites. Also, the conversion could have been affected by the lower pore volume of LZ-100 (Table 1). The catalytic evaluation of toluene disproportionation over ZSM-5 with various crystal sizes $(0.5,5,50$ and 100$) \mu \mathrm{m}$ at WHSV $3 \mathrm{~h}^{-1}$ is shown in Figure 6. In the graphs, time zero minutes is defined as the appearance of the first liquid product drop from the separator sampling point which usually takes two hours after starting the 
feed pump. The difference in crystal size, imposed diffusion limitation and catalyst acidity resulted in different toluene conversions by small and large crystals.
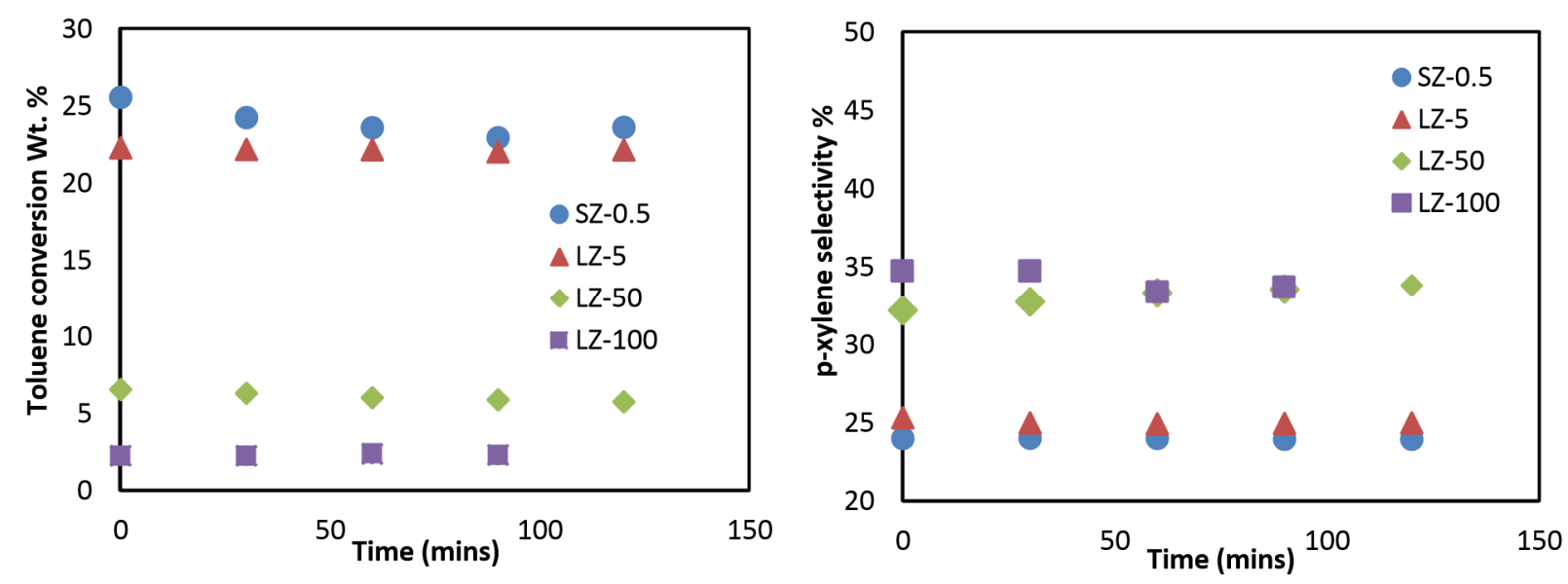

Figure 6: Effect of crystal size on p-xylene selectivity and toluene conversion

\subsubsection{Effect of space velocity (WHSV)}

Increasing the space velocity leads to a reduced the contact time between the the catalyst and reactants/products. Thus, it was observed that increasing WHSV resulted in lower conversion. Alternatively, para-selectivity was noticeably enhanced reaching its highest value at the fastest WHSV $\left(83 \mathrm{~h}^{-1}\right)$ on all catalysts as seen in Figure 7. The selectivity was not much improved by LZ-5 at low flowrates (WHSV). However, there was an improvement in selectivity when increasing the flowrate (WHSV) which can be observed with LZ-5 while SZ-0.5 did not affect the selectivity even at a very high flowrate. This indicates that the larger crystal size contributed to the increase in p-xylene selectivity. Also, it should be taken into consideration that LZ-5 has higher acidity which could conversely affect the selectivity. Thus, reducing the acidity LZ-5 with various modifications techniques could lead to even more improvement in the selectivity of $p$ xylene. The selectivity obtained with LZ-5 at high WHSV $\left(83 \mathrm{~h}^{-1}\right)$ was about $45 \%$. On the other hand, SZ-0.5 delivered a selectivity around $27 \%$ which is close to the thermodynamic equilibrium value. The highest p-xylene selectivities were attained by LZ-50 and LZ-100 at all WHSVs. However, the obtained toluene conversions were the lowest amongst the other catalysts. The 
optimum results in terms of the combination of toluene conversion, pxylene selectivity and $p$ xylene yield were achieved over LZ-5. Based on the achieved results it can be concluded that crystal size plays an important role in enhancing the p-xylene selectivity in the xylene mixture. Moreover, greater diffusion limitations are imposed on the isomers with larger kinetic diameters by increasing the diffusion pathway (crystal size). Therefore, they will isomerize to the favourable para isomer, which have a smaller kinetic diameter, in order to diffuse out of the channels. Similar results were obtained at atmospheric pressure in the literature and are in agreement with the findings of this study [29]. Furthermore, the highest yield of $p$-xylene was achieved over LZ-5 as a result of the high conversion delivered at all WHSVs except for WHSV $3 \mathrm{~h}^{-1}$ (Figure 8).
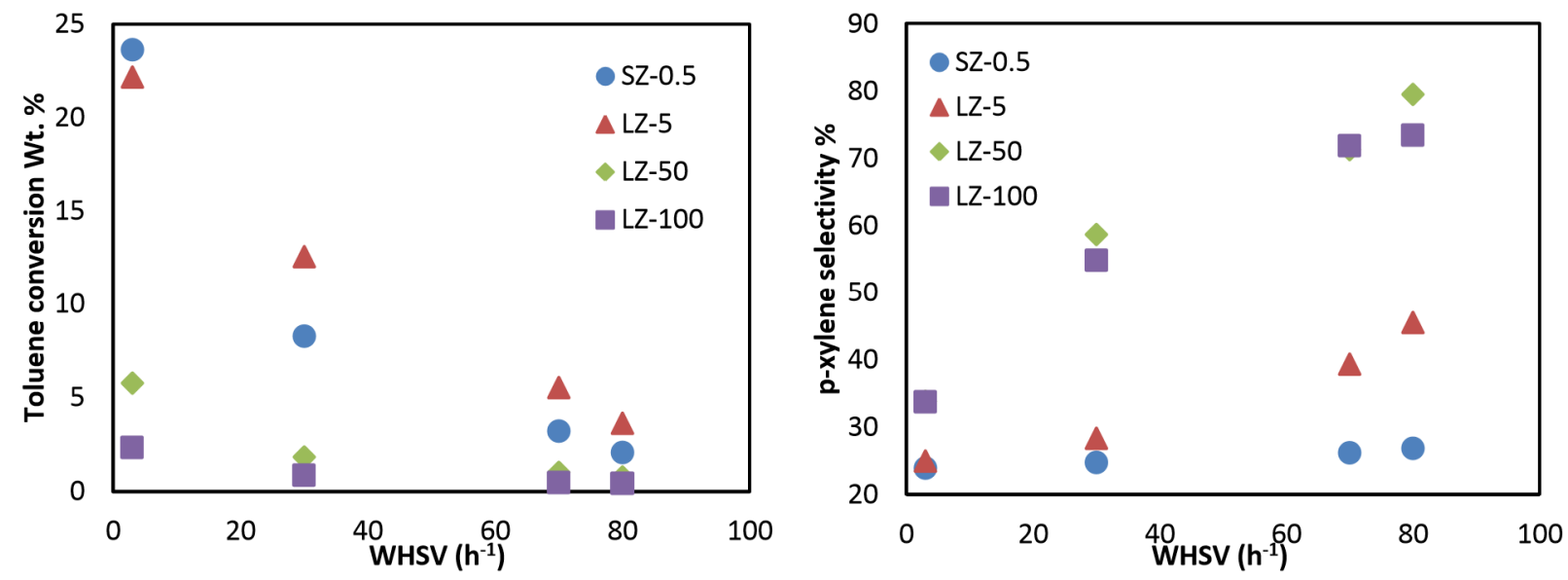

Figure 7: Effect of WHSV $\left(\mathrm{h}^{-1}\right)$ on $\mathrm{p}$-xylene selectivity and toluene conversion 


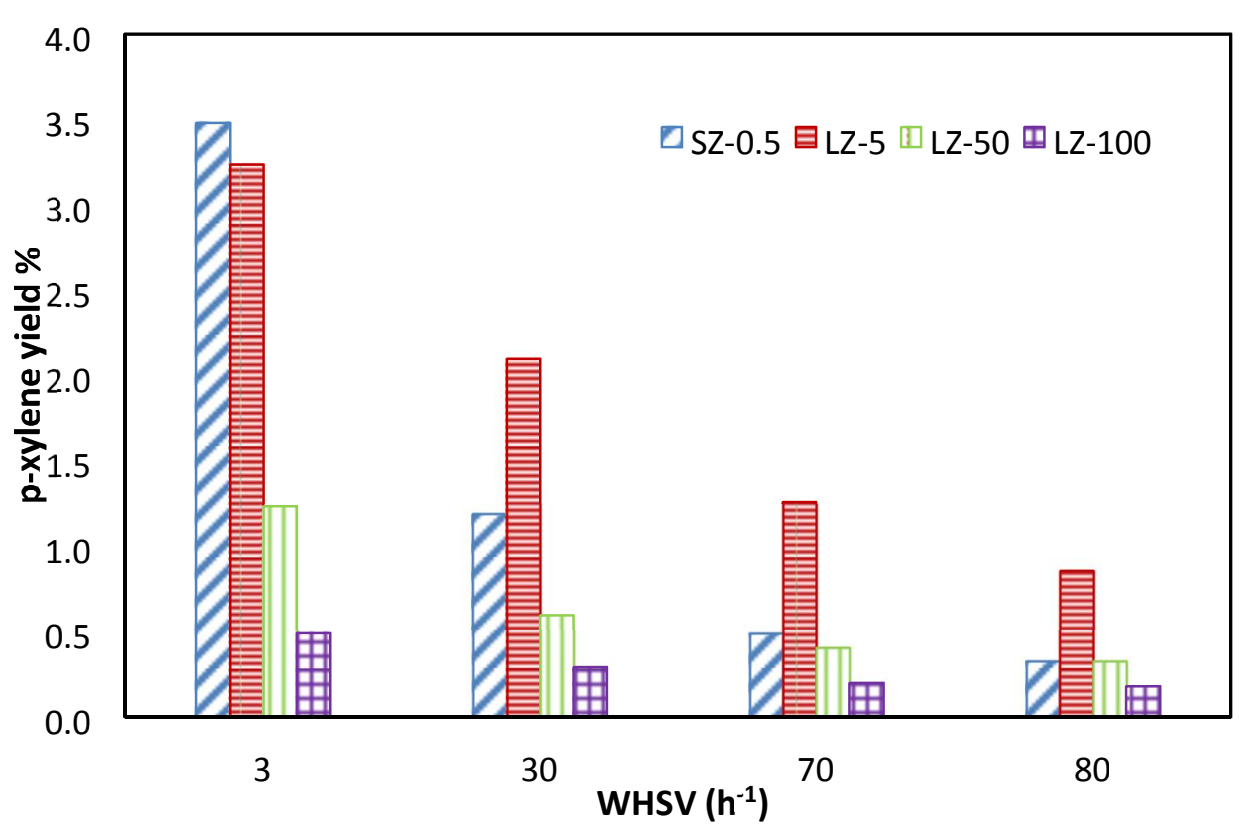

Figure 8: Effect of crystal size at different WHSVs on $p$-xylene yield

\subsubsection{Effect of elevated pressure}

Increasing the pressure allows for more and longer contact between the toluene molecules and the zeolite. Therefore, higher chance of feed molecules reacting and converting into products on the available acid sites within ZSM-5. Increasing the pressure to 10 bar with hydrogen, significantly increased in the conversion of toluene. The effects of raising the pressure to 10 bar can be observed clearly in Figure 9 compared to the results at 1 bar in section 3.2.2. It was noticed that carrying out the reaction at high pressure improved the toluene conversion. It can also be observed that in the case of large crystals catalysts, the selectivity towards p-xylene was increased with increasing WHSV $\left(h^{-1}\right)$. However, it is lower than the attained values at atmospheric pressure. The obtained results indicated a trade-off between conversion and selectivity.

LZ-5 produced the highest conversion at about $46 \%$ at the lowest WHSV. This achieved conversion can be ascribed to the more available acid sites (Table 1) that can be utilized in the reaction on this catalyst. This allowed LZ-5 to perform better in terms of toluene conversion even when the WHSV was varied from $\left(3\right.$ to $83 \mathrm{~h}^{-1}$ ). LZ-50 and LZ-100 delivered the highest pxylene 
selectivity $50 \%$ and $60 \%$, respectively at the highest space velocity. On the other hand, they showed the lowest conversion which was around $5 \%$ and $2 \%$, respectively.
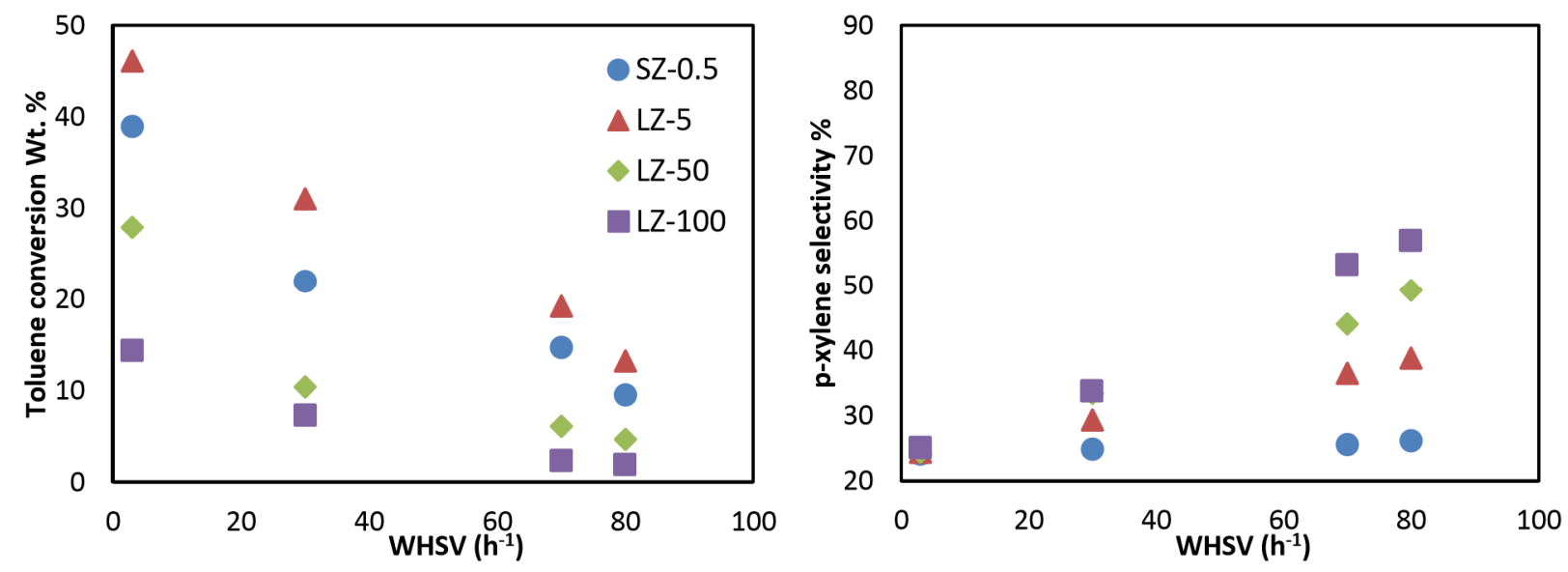

Figure 9: Effect of pressure on conversion and p-xylene selectivity

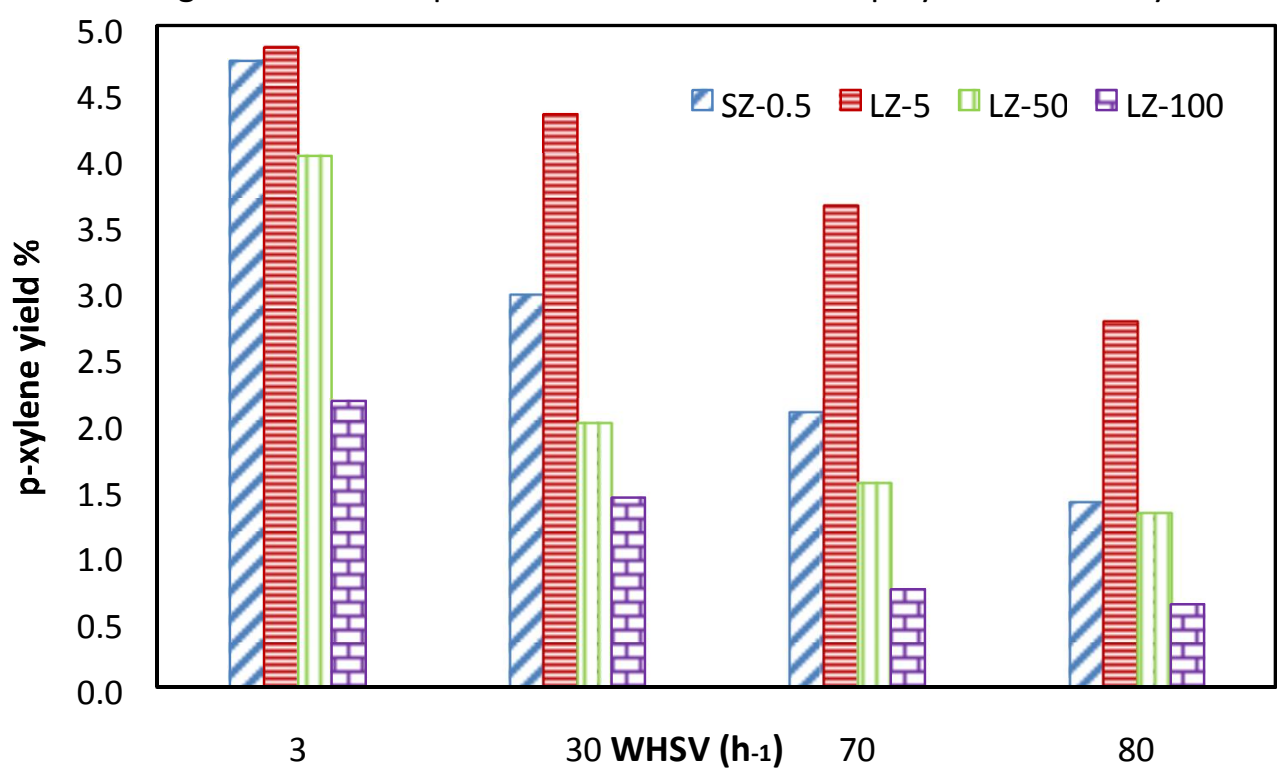

Figure 10: p-xylene yield at 10 bar over ZSM-5 with a range of crystal size

\subsubsection{Deactivation behaviour of ZSM-5 with different crystal sizes at 10 bar}

The performance of SZ-0.5, LZ-5 and LZ-100 was evaluated for 50 hours on stream at a WHSV $30 \mathrm{~h}^{-1}$ to investigate the effect of crystal size on toluene conversion and $\mathrm{p}$-xylene selectivity over extended operation (Figure 11). It is observable from the graph that the conversion decreased with time over all three catalysts. The conversion over the large crystals (LZ-5 and LZ100) decreased rapidly initially and then more slowly after 12 hours. On the other hand, the 
smaller crystal size continued to deactivate up to 40 hours and then stabilized. The selectivity

towards p-xylene was significantly improved with time over LZ-100 and increased from around $34 \%$ to $55 \%$ after 30 hours on stream. Conversely, SZ-0.5 did not improve the selectivity towards p-xylene which remained around the thermodynamic equilibrium value during the whole run. Likewise, only minor improvement of the selectivity was observed over LZ-5 reaching $27.5 \%$ after 50 hours on stream. Similar behaviour over ZSM- 5 was observed by

Velasco et al. [30]. However, their observation was obtained from ethylbenzene disproportionation over ZSM-5 (Si/Al $=42$ and crystal size of $4.4 \mu \mathrm{m}$ ) at $300^{\circ} \mathrm{C}$ and atmospheric pressure. They found that there was a fast deactivation during the first three hours on stream. The amount of coke accumulated on all three catalysts was determined using TGA. The highest amount of coke was found on LZ-100 while the lowest amount was formed over ZSM-5 with the smallest crystal size (SZ-0.5). This could be a result of the higher BET surface area and shorter diffusion path which allows faster diffusion of larger molecules formed inside the channels. Furthermore, the high amount of coke formed on the large crystals LZ-100 could be a result of the observed low pore volume (Table 1). Wan et al. reported similar results confirming that the longer the diffusion path the more observed coke accumulation on the catalyst. This is due to that the large molecules will have difficulty diffusing out leading to the formation of bulkier molecules and eventually coke [31]. Figure 12 shows the TGA weight loss curves of all spent catalysts as weight percent after performing toluene disproportionation for 50 hours at a WHSV $30 \mathrm{~h}^{-1}$. 

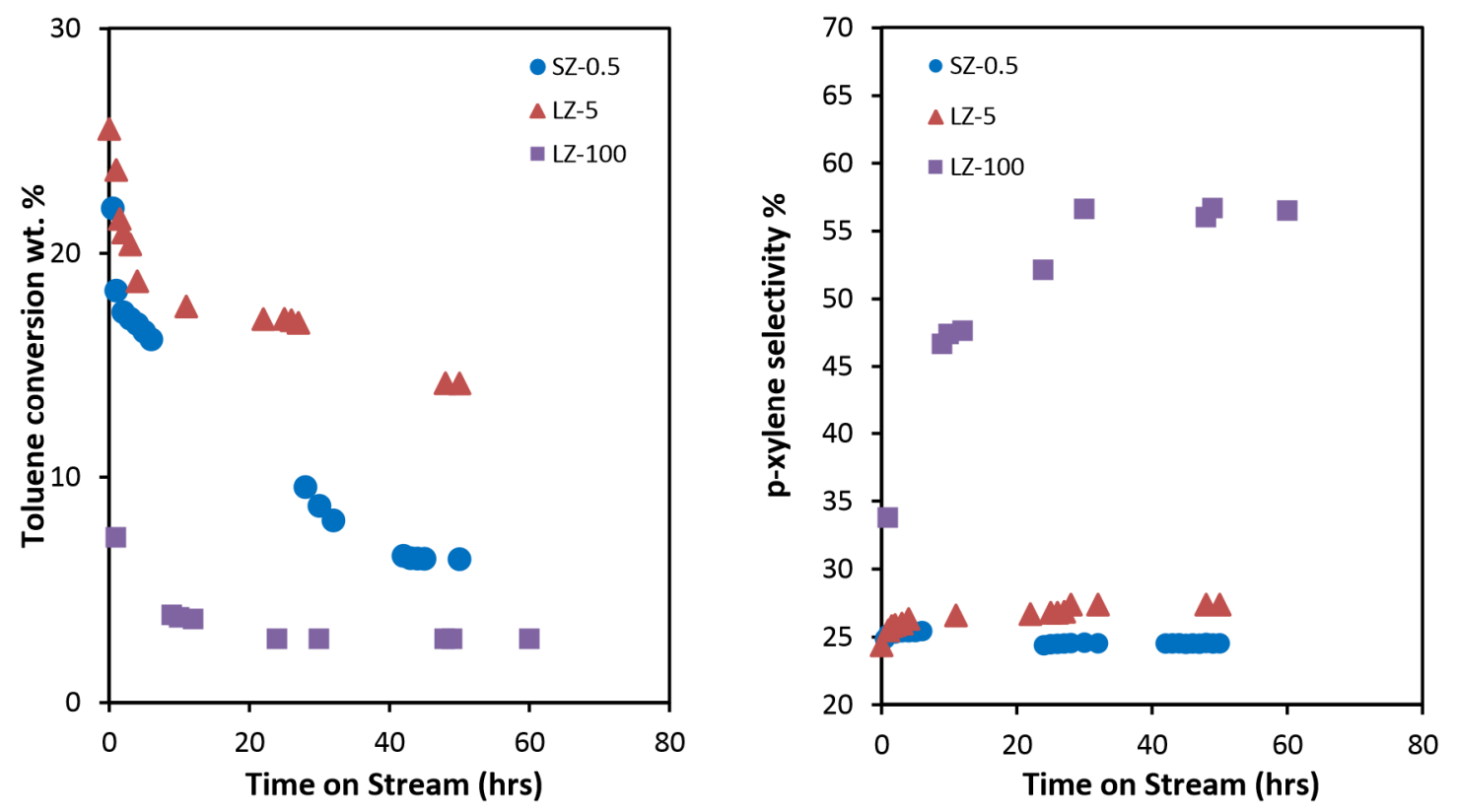

Figure 11: Deactivation behaviour over ZSM-5 zeolites with different crystal size

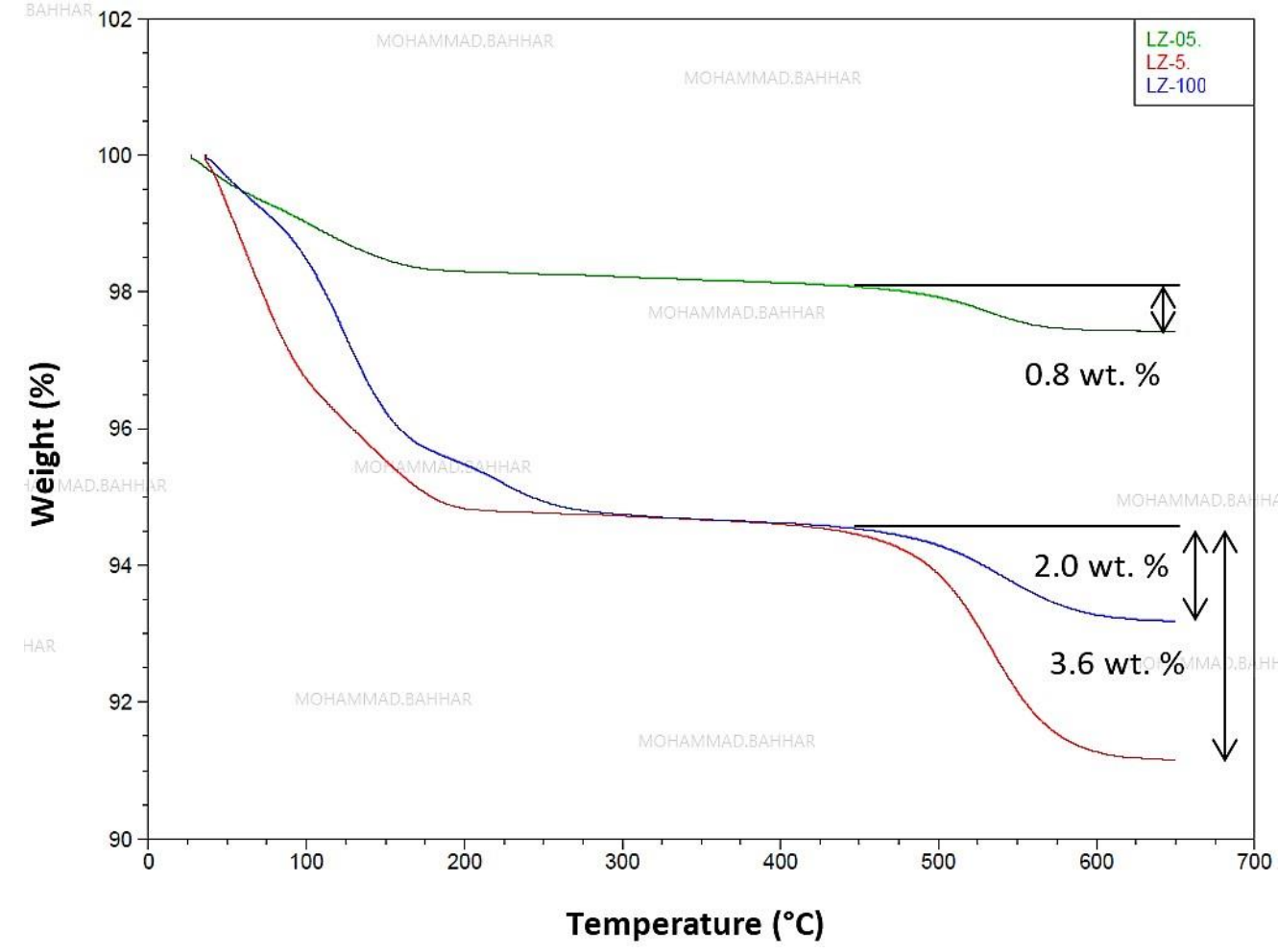

Figure 12: TGA curves for ZSM-5 with different crystal sizes after 50 hours on stream

Table 2 shows the weight loss for all zeolites and the lowest amount of coke was obtained by the smallest crystal size $(0.5 \mu \mathrm{m})$. Moreover, LZ-5 $(5 \mu \mathrm{m})$ had the largest amount of coke as it has more acid sites (Table 1) than LZ-100 (100 $\mu \mathrm{m})$.

Table 2: Coke amount after deactivation studies 


\begin{tabular}{lcccc}
\hline Catalyst & Si/Al & X (50h) & Sp $(50 \mathrm{~h})$ & Coke wt. \% \\
\hline LZ-0.5 & 26.3 & 7.2 & 24.5 & 0.80 \\
LZ-5 & 15.9 & 14.2 & 27.5 & 3.61 \\
LZ-100 & 64.3 & 2.8 & 56.7 & 2.00 \\
\hline
\end{tabular}

\section{Conclusion}

Toluene disproportionation was performed over four ZSM-5 zeolites synthesized in house with various crystal sizes $(0.5,5,50$ and $100 \mu \mathrm{m})$ to examine the effect of crystal size on toluene conversion and p-xylene selectivity. Furthermore, the effects of several key operating parameters including reaction pressure and space velocity were studied to optimize the conversion and selectivity of toluene disproportionation. Increasing the crystal size resulted in the improvement in p-xylene selectivity and oppositely the conversion was decreased. The high selectivity to p-xylene was especially attained with the very large crystals $(50,100) \mu \mathrm{m}$. Moreover, increasing the space velocity (WHSV) furtherly enhanced the selectivity towards pxylene and decreased the conversion. The increase in selectivity was observed only with ZSM-5 with large crystals $(5,50$, and 100$) \mu \mathrm{m}$ indicating that the obtained improvement is due to the combination of increasing the crystal size and space velocity. Alternatively, increasing the pressure from atmospheric to 10 bar improved the toluene conversion at the cost of $p$-xylene selectivity. The highest conversion was achieved over the $5 \mu \mathrm{m}$ (LZ-5) while the $100 \mu \mathrm{m}$ (LZ-100) delivered the highest $\mathrm{p}$-xylene selectivity accompanied by a low conversion. The study concluded that LZ-5 achieved the best results in terms of p-xylene selectivity and conversion combination. Figure 13 summarizes toluene conversion and p-xylene selectivity at 10 bar for all evaluated catalysts. 


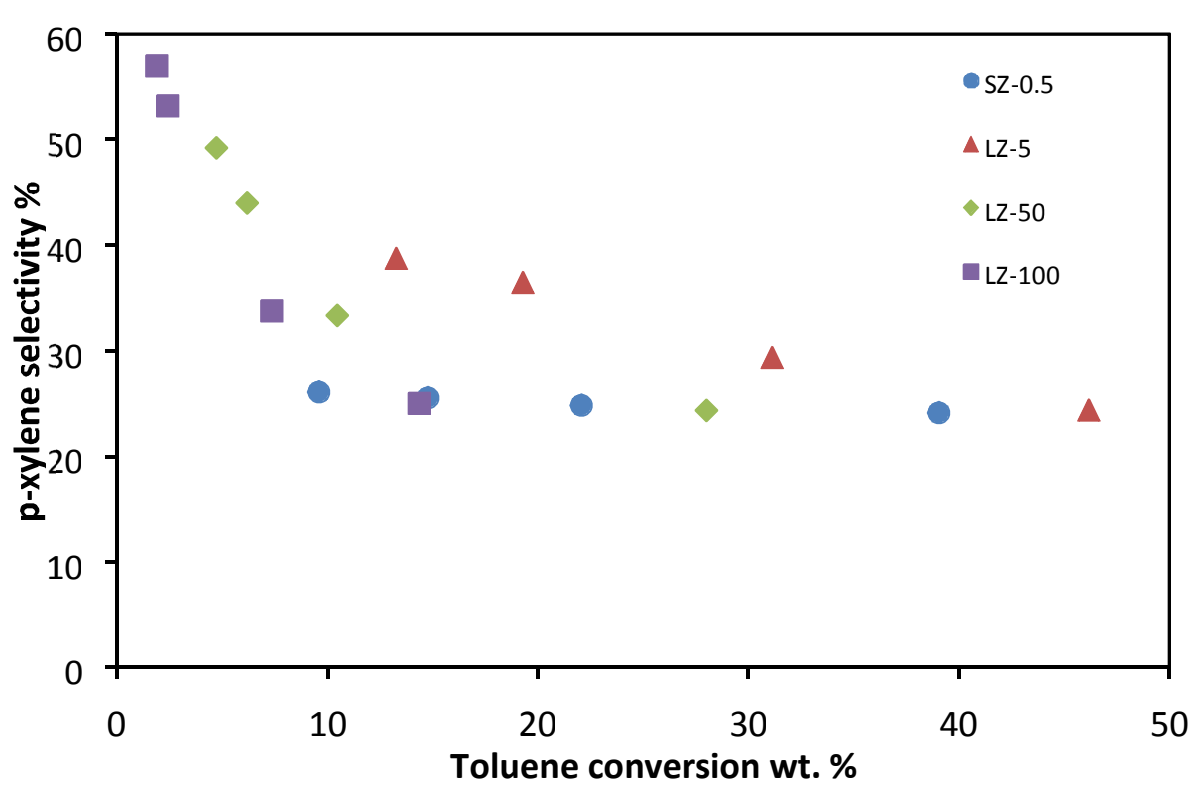

Figure 13: Toluene conversion vs p-xylene selectivity over ZSM-5 with different crystal sizes

This research presents unique findings where different crystal sizes were applied and it showed that even though increasing the diffusion path by increasing the crystal size is an important factor that size should be tailored during the synthesis to a certain range. It is demonstrated throughout this paper that crystal size between 5-7 $\mu \mathrm{m}$ is not enough and the diffusion pathway has to be long enough to restrict the diffusion of the undesired xylene isomers. It was observed that a crystal size around $50 \mu \mathrm{m}$ enhanced the selectivity significantly. Moreover, increasing the crystal size further does not improve the p-xylene selectivity much further. Increasing the crystal size further will instead decrease the conversion as the diffusion path is too long which will restrict larger molecule to diffuse out and eventually results in deactivating the catalyst. Moreover, it was also concluded that during synthesis of larger crystals than $50 \mu \mathrm{m}$, aluminium is difficult to incorporate in the structure resulting in crystals with lower acidity.

Finally, increasing the diffusion limitation by increasing the diffusion path length (crystal size) is a key factor in enhancing the selectivity towards p-xylene. To furtherly optimize the selectivity, the quick isomerization reaction of xylene over the external surface of zeolites should be suppressed through deactivating the external acid sites. 
Acknowledgments: The authors are thankful for the financial support from Saudi Aramco. Also, would like to acknowledge the continuous lab technical support from Mr. Loris Doyle and Ms. Shahla Khan. Finally, we would like to acknowledge and thank Dr. John Waters for his support with BET analysis.

\section{References}

1) T. Tsai, Appl. Catal. A., 182 (1999), P. 355.

2) W. Vermeiren, J.P. Gilson, Top Catal., 52 (2009), P. 1131.

3) S. Jenkins, [Presentation] IOC Petrochemical conclave. (2013) http://www.petrochemconclave.com/presentation/2013/Mr.SJenkins.pdf (accessed 15 September 2019)

4) K. Tominaga, S. Maruoka, M. Gotoh, N. Katada, M. Niwa, Microporous Mesoporous Mater., 117(2009), p. 523.

5) D.B. Shah, C.J. Guo, D. T. Hayhurst, Stud. Surf. Sci. Catal., 80 (1993), P. 575.

6) D. Mitsuyoshi, K. Kuroiwa, Y. Kataoka, T. Nakagawa, M. Kosaka, K. Nakamura, S. Suganuma, Y. Araki, N. Katada, Micropourous Mesoporous Mater., 242 (2017), P. 118.

7) N.Y. Chen, W.W. Kaeding, F.G. Dwyer, J. Am. Chem. Soc., 101 (1979), P. 6783.

8) W.W. Kaeding, C. Chu, L. B. Young, S. A. Butter, J. Catal., 69 (1981) P. 392.

9) L. B. Young, S. A. Butter, W. W. Kaeding, J. Catal., 76 (1982), P. 418.

10) P. Ratnasamy, G. P. Babu, A. J. Chandwadkar, S. B. Kulkarni, Zeolites., 6 (1986) P. 98.

11) V. Ducarme, J. Vedrine. Appl. Catal., 17 (1985), P. 175.

12) F. Bauer, W. Chen, Q. Zhao, A. Freyer, S. Liu, Microporous Mesoporous Mater., 47(2001), P.67.

13) P. Wu, T. Komatsu, T. Yashima, Microporous Mesoporous Mater., 22(1998), P. 343.

14) Z. Zhu, Z. Xie, Q. Chen, D. Kong, W. Li, W. Yang, C. Li, Microporous Mesoporous Mater., 101(2007), P. 169.

15) R. Le Van Mao, V. Ragaini, G. Leofanti, R. Fois, J. Catal., 81 (1983), P. 418.

16) F. Bauer, E. Bliz, A. Freyer, Stud. Surf. Sci. Catal., 154 (2004), P. 2169.

17) D. Olson, W. Haag, ACS. Symp. Ser. Am. Chem. Soc., 248 (1984), P. 275. 
18) S. Al-Khattaf, Chem. Eng. Process, 46 (2007), P. 964.

19) H. Wu, M. Liu, W. Tan, K. Hou, A. Zhang, Y. Wang, X. Guo, J. Energy Chem., 23 (2014), P. 491.

20) J. Hodala, A. Halgeri, G. Shanbhag, Appl. Catal. A. 484 (2014), P. 8.

21) Y. S. Bhat, J. Das, K. V. Rao, A. B. Halgeri, J. Catal., 159 (1996), P. 368.

22) M. Uguina, J. Sotelo, D. Serrano, Appl. Catal., 76 (1991), P. 183.

23) N. Chen, J. Catal., 114 (1988) P. 17.

24) C. Rallan, R. Al-Rubaye, A. Garforth, Chem. Eng. Trans., 43 (2015), P. 907.

25) S. Z. Chen, K. Huddersman, D. Keir, L.V. C Rees, Zeolites, 8 (1988), P. 106.

26) E. Derouane, J. Védrine, R. Pinto, P. Borges, L. Costa, A. Lemos, Catal. Rev., 55 (2013), P. 454.

27) U. Müller, K. Unger. Stud. Surf. Sci. Catal., 39 (1988), P.101

28) S. Lowell, J. E. Shields, M. A. Thomas, M. Thommes. Characterization of Porous Solids and Powders: Surface Area, Pore Size and Density. Kluwer Academic Publishers, Dordrecht, the Netherlands, 2004.

29) N. Chen. Ind. Eng. Chem. Res., 40 (2001), P. 4157.

30) N. Velasco, M. Machado, D. Cardoso, Braz. J. Chem. Eng., 15 (1998).

31) Z. Wan, C. Wang, H. Yang, D. Zhang, In Chemeca 2013 : challenging tomorrow. Vol. 1. Barton, ACT: Engineers Australia, (2013), P. 5. 
